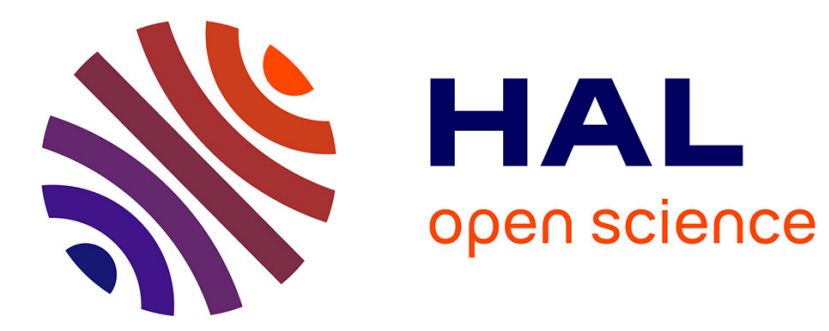

\title{
Effets de l'irradiation aux UV sur les propriétés mécaniques des matériaux composites polymères
}

Aurélie Cordelle, Monssef Drissi-Habti, Aaron Forster, Joannie Chin

\section{To cite this version:}

Aurélie Cordelle, Monssef Drissi-Habti, Aaron Forster, Joannie Chin. Effets de l'irradiation aux UV sur les propriétés mécaniques des matériaux composites polymères. Revue des composites et des matériaux avancés = Journal of Composite and Advanced Materials, 2013, 23 (2), pp. 295-309. hal00867235

\section{HAL Id: hal-00867235 https://hal.science/hal-00867235}

Submitted on 27 Sep 2013

HAL is a multi-disciplinary open access archive for the deposit and dissemination of scientific research documents, whether they are published or not. The documents may come from teaching and research institutions in France or abroad, or from public or private research centers.
L'archive ouverte pluridisciplinaire HAL, est destinée au dépôt et à la diffusion de documents scientifiques de niveau recherche, publiés ou non, émanant des établissements d'enseignement et de recherche français ou étrangers, des laboratoires publics ou privés. 


\title{
Effets de l'irradiation aux UV sur les propriétés mécaniques des matériaux composites polymères
}

\author{
Aurélie CORDELLE ${ }^{*}$ - Monssef DRISSI-HABTI ${ }^{* \alpha}$ - Aaron \\ FORSTER $^{* *}$ - Joannie CHIN ${ }^{* * *}$
}

* PRES LUNAM, IFSTTAR, Département Mesure,

Auscultation et Calcul Scientifique (MACS)

44344 Bouguenais Cedex, FRANCE

a Pour toute correspondence : monssef.drissi-habti@ifsttar.fr

* NIST, Materials and Construction Research Division

Building and Fire Research Laboratory

Gaithersburg, MD, Etats-Unis

\begin{abstract}
RÉSUMÉ. Afin de convaincre les industriels pour une introduction massive des matériaux composites dans des constructions exposées aux intempéries, les performances de ces matériaux doivent demeurer les meilleures possibles, mêmes après des expositions prolongées en environnement extérieur, sous température, humidité et rayonnement solaire. A l'aide d'une installation spécifique, des échantillons en résine vinylester monolithique, ainsi qu'en matériau composite vinylester/fibre de verre ont été exposés à un rayonnement $U V$, très dense. Afin d'évaluer l'impact de cette exposition aux UV, les techniques de nanoindentation instrumentée et de microscopie confocale ont été utilisées. Cette dernière a permis la visualisation $3 D$ et la quantification de la rugosité du vinylester due à l'exposition aux UV alors que la technique de nanoindentation permet de mesurer le module d'Young local et la dureté, sur la surface exposée. Sur les échantillons exposés aux UV, une surface très escarpée a été obtenue et dans ces circonstances, l'hypothèse d'une pression hydrostatique lors de l'essai de nano-indentation ne peut plus être vérifiée. Le but de cette étude est de mettre en œuvre une technique d'investigation contournant ce problème.

ABSTRACT. The use of polymer matrix composites in civil engineering and construction (including offshore) is becoming increasingly attractive. Therefore, the mechanical performance as a function of outdoor environment, particulary after high intensity $U V$ exposures, is becoming a real issue. In this work, samples made out of monolithic vinylester as well as vinylester/glass-fiber composite were exposed to UV-radiation. The mechanical behaviour of these samples was characterized mainly using nanoindentation. Laser Scanning Confocal Microscopy (LSCM) was used to quantify the topography of the samples that were roughened following $U V$-exposure. A method is herein developed and is based on a cross-cut of samples and a polish of the transverse face providing a mirror finish needed for nanoindentation while avoiding the elimination of the exposed surface. This method is enabling the estimate of the local Young's modulus and hardness as a function of exposure time, while specifying the thickness of the material affected by $U V$.
\end{abstract}


MOTS-CLÉS : dégradation UV, nanoindentation, microscope confocal, résine vinylester. KEYWORDS: UV-degradation, nanoindentation, confocal microscope, vinylester resin.

\section{Extended abstract}

Composite materials are challenging materials for use as structural parts in civil engineering and construction. Their main advantages are pertaining to their excellent corrosion resistance and strength-to-weight ratio, high stiffness, relatively good resistance to environmental agents and fatigue, good processability and ease of installation. These advantages have led them to be considered over more conventional materials such as steels and reinforced concrete. Polymer composite materials can be applied for rehabilitation of structural components systems. They also have shown promising potential as construction materials in new infrastructure components.

However, despite the substantial advantages of composites, some critical issues remain regarding their environmental durability and long-term performance. It should be noted that previous research on thermosetting resins, glass fibers and their composites has shown significant losses in their mechanical properties and integrity when exposed to aggressive conditions for prolonged periods of time, such as the Hygrothermal Exposure and Sustain Strain [1]. Indeed the outdoor environment can be detrimental to organic polymers: moisture, acid rain, temperature cycling and ultraviolet $(U V)$ radiation can lower the overall performance of a polymer.

One of the main performance that outdoor composite applications should exhibit is the ability to withstand long term exposure without any mechanical properties loss. Attempts at linking field and laboratory exposure results and at predicting the service life of a polymeric material exposed in its service environment have been a high priority research topic of the polymeric materials community for over a century [2]. The inability to generate accurate, precise, and timely service life estimates for polymeric systems exposed in their intended service environments hinders product innovation as well as the timely introduction of new polymeric materials into the market.

At present, the only accepted way of generating a performance history for a new product is to expose it for many years at one or more "standard" field exposure sites such as Florida or Arizona. Performance data generated from such experiments, however, are neither repeatable nor reproducible, since the weather never repeats itself over any time scale or at any location [2]. Laboratory weathering experiments are typically designed to simulate and accelerate outdoor degradation by exposing materials for extended periods of time to high UV irradiance, elevated temperature, and relative humidity environments. However, the lack of rigorous temporal and spatial experimental control over each of the weathering factors comprising an exposure environment within and among laboratory exposure devices has made it impossible to correlate results between laboratory devices and to link laboratory and field exposure data.

A French national project aiming at erecting a large dimensions platform entirely made out of smart composites (DECID2) has been approved in 2008. A 
composite combination based on E-glass fibers and vinylester matrix was chosen as constituent materials. Among the critical issues of the project is the assessment of the mechanical properties of composites that have undergone heavy uv radiation exposure. Degradations were characterized mainly using nanoindentation testing and Laser Scanning Confocal Microscopy (LSCM). The mechanical characterization based on macro-mechanical testing is therefore not adapted, UV radiation affects a thin layer of composite surface, only nanoindentation have shown proof of reliability in assessing such changes.

\section{Introduction}

Les matériaux composites polymères sont de plus en plus utilisés pour la réhabilitation des systèmes de composants structuraux dans le génie civil. Ils ont également montré un potentiel prometteur en tant que matériaux de constructions (y compris dans les constructions maritimes). Leurs principaux avantages consistent en : leurs excellentes résistances mécanique et à la corrosion, leur rapport masse/rigidité élevé, leur bonne aptitude au traitement et leur facilité d'installation. Ces avantages ont amené à prendre en considération l'utilisation potentielle de ces derniers en remplacement des matériaux plus conventionnels tels que les aciers et le béton armé, dans certaines applications. Cependant, malgré ces avantages considérables, certaines questions essentielles demeurent quant à leur durabilité et leurs performances environnementales sur le long terme. En effet, l'environnement extérieur peut être préjudiciable : l'humidité (Helbling et al., 2008), les pluies acides, les cycles de température et surtout ce qui est l'objet de cette étude, les rayons ultraviolets (UV) peuvent réduire la performance globale d'un polymère et/ou des fibres (Martin et al., 2005).

Dans cette étude, les échantillons sont exposés dans la SPHERE (Simulated Photodegradation via High Energy Radiant Exposure, NIST, Gaithersburg, Md, Etats-Unis). L'utilisation de la nanoindentation permet une caractérisation mécanique locale des propriétés de ces matériaux. Cependant, afin de se placer dans l'hypothèse de contrainte hydrostatique, la surface indentée doit être polie, du type miroir. Or, la mise en évidence d'une forte rugosité, comme conséquence de l'exposition aux UV, est un problème réel qu'il faudrait contourner car cette surface rugueuse contient toutes les informations que nous souhaitons exploiter. Le challenge de cette étude est de proposer une méthode contournant ce problème de rugosité, sachant qu'il est impossible de polir la surface exposée au risque d'éliminer la couche à analyser.

\section{Matériaux \& Méthodes}

\subsection{Préparation de l'échantillon}

Les échantillons de vinylester ainsi que ceux de composites fabriqués par pultrusion (fraction volumique des fibres de verre de $66 \%$ et une matrice vinylester) sont découpés à l'aide d'une scie diamantée et polis avec du papier abrasif 600, 800 
et 1200, puis avec des solutions diamantées dont les tailles de grain font respectivement $15,9,6,3,1$ et $0,05 \mu \mathrm{m}$. Pour étudier les sections transversales, les échantillons sont découpés transversalement, enrobés et polis selon le même protocole.

\subsection{Exposition aux rayons $U V$ (Simulated Photodegradation via High Energy Radiant Exposure, SPHERE)}

Développée au NIST (Gaithersburg, Md, Etats-Unis), la SPHERE fournit une source de rayonnement UV de longueur d'onde compris entre $290 \mathrm{~nm}$ à $400 \mathrm{~nm}$ (Brown et al., 2000). L'exposition a été réalisée dans des conditions extrêmes : les échantillons ont été exposés initialement à $55^{\circ} \mathrm{C}$, avec une humidité de $75 \%$ pendant environ 2 jours, puis la température a été diminuée à $35^{\circ} \mathrm{C}$ et au bout de 4 jours, l'humidité a été diminuée à $50 \%$ et ceci jusqu'à la fin des essais. Le flux reçu est d'environ $150 \mathrm{~W} / \mathrm{m}^{2}$. Une série d'échantillons a été retirée de la SPHERE après 2 semaines d'exposition et une seconde a complété 4 semaines. La troisième série n'a pas reçu d'exposition aux UV et a servi de comparatif.

\subsection{Morphologies de surfaces}

La morphologie de surface est caractérisée par un microscope confocal du type Zeiss LSM510, qui permet également de mesurer la rugosité de l'échantillon. Cette technique utilise une lumière cohérente $(\mathrm{HeNe}, 543 \mathrm{~nm})$ et recueille la lumière exclusivement du plan focal, tout en rejetant celle hors de ce plan. Les images sont traitées à l'aide du logiciel. En déplaçant le plan focal, les images individuelles (tranches optiques) peuvent être combinées pour construire une image en trois dimensions.

\subsection{Nanoindentation}

Contrairement au test statique de macro et micro dureté, la nanoindentation instrumentée a, non seulement une très haute résolution spatiale, mais fournit également, en temps réel, les données de force et de pénétration lors du chargement et du déchargement (Figure 1). Lorsque l'indenteur pénètre dans le matériau, des déformations élastiques et plastiques provoquent la formation d'une impression de dureté. Lors du retrait de la pointe, seule la partie élastique du déplacement est récupérée. C'est cette récupération qui permet de déterminer les propriétés élastiques (Cook et al., 2007). La surface de contact permet ainsi d'évaluer les propriétés de dureté (Fischer-Cripps, 2004). 

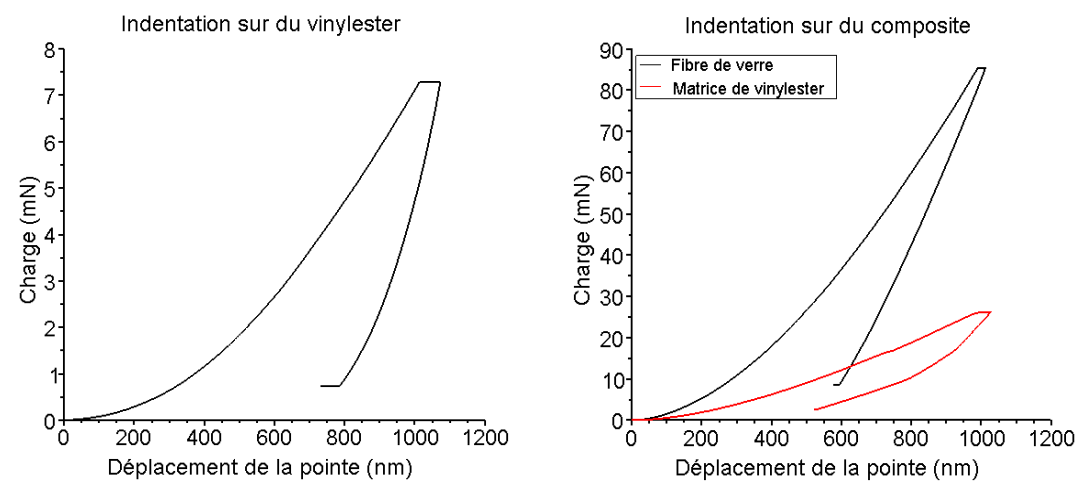

Figures 1. Charge en fonction du déplacement sur du vinylester seul et sur du composite : indentation sur la fibre de verre et indentation sur la matrice de vinylester.

Pour les calculs suivants, nous limitons la discussion à un matériau continu et isotrope, dont les dimensions sont très grandes par rapport à la profondeur de l'indentation. Les surfaces sont considérées lisses et le contact se fait sans frottement. En outre, nous supposerons qu'il n'y a pas de dépendance au temps et absence de fissuration au cours de l'indentation (ceci est d'ailleurs confirmé par observation en microscopie). Enfin, les résultats ne peuvent être exploités que si la contrainte exercée est hydrostatique. Dans ces conditions, la dureté et le module d'Young local peuvent être définis à l'aide des équations [1] à [3] :

$$
\begin{aligned}
& H=\frac{P}{A} \\
& E_{r}=\frac{\sqrt{\pi} S}{\beta 2 \sqrt{A}} \\
& \frac{1}{E_{r}}=\frac{\left(1-v_{s}^{2}\right)}{E_{s}}+\frac{\left(1-v_{i}^{2}\right)}{E_{i}}
\end{aligned}
$$

Où, $\mathrm{H}$ est la dureté, $\mathrm{P}$ la charge, A la surface de contact, Er le module réduit, $\mathrm{S}$ la rigidité, $\beta$ un facteur correctif de la pointe $(\beta=1$ pour un contact circulaire, $\beta=1.034$ pour une pyramide de Berkovich), Es et Ei les module de l'échantillon et de l' 'indenteur, vs et vi sont le coefficient de Poisson de l'échantillon et de l'indenteur.

Les mesures ont été effectuées en utilisant le nanoindenteur MTS Nanoinstruments, NanoXP. Les résolutions de charge et de déplacement sont de 50 $\mathrm{nN}$ et $0,04 \mathrm{~nm}$, respectivement. Deux types d'indenteurs ont été utilisés afin de comparer et valider les résultats quelque soit la forme de l'indentation: un indenteur 
sphérico-conique de rayon de la sphère, $10 \mu \mathrm{m}$ et d'angle du cône, $90^{\circ}$ et un indenteur pyramidal, du type Berkovich. Les résultats sont similaires mais l'indenteur Berkovich est préféré pour une meilleure visualisation de l'indentation sous microscopie confocale (Figure 2). La calibration de l'indenteur est effectuée en utilisant les caractéristiques connues de la silice fondue. Le chargement a été effectué à une vitesse de déformation constante de $0,05 \mathrm{~s}-1$. Les profondeurs utilisées pour cette étude sont de 500, 750 et $1000 \mathrm{~nm}$. Pour éviter toutes interactions entre elles, les indentations sont espacées d'au moins 50 microns.

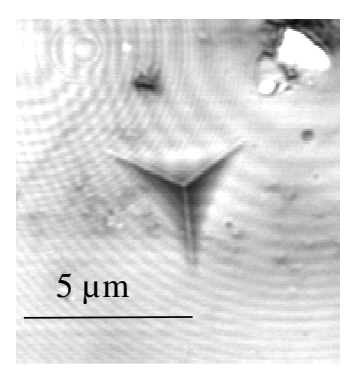

Figure 2. Micrographie 2D, empreinte d'une indentation avec un indenteur de Berkovich.

Deux méthodes sont utilisées pour déterminer la rigidité : la méthode du déchargement, où la rigidité correspond à la pente à l'origine de la courbe de déchargement et la méthode «continous stiffness measurment CSM (Oliver et al. 1992). Cette dernière technique consiste à ajouter des faibles oscillations (dans le cadre de ce travail, $5 \mathrm{~nm}$ ) pendant la charge à une fréquence élevée (45 Hz). La raideur du contact est mesurée par la réponse du déplacement à la fréquence d'excitation. Cette technique permet d'évaluer le module et la rigidité en fonction de la profondeur de pénétration, les caractéristiques mécaniques sont déduites lorsqu'elles ont atteint une valeur palier après une pénétration d'environ $200 \mathrm{~nm}$. Nous devons garder à l'esprit que pendant les premières oscillations le comportement de plasticité locale sous l'indenteur n'est pas encore atteint. Une fois la plasticité locale atteinte, la réponse élastique au moment du déchargement conduit à une estimation pertinente des propriétés mécaniques du matériau testé, se traduisant par l'atteinte d'une valeur plateau (Drissi-Habti et al., 1998).

\section{Résultats et discussions}

\subsection{Analyse microstructurale}

Avant de mesurer les propriétés mécaniques, l'utilisation de la microscopie confocale permet la visualisation des surfaces, exposées ou non, et fournit des 
informations sur la structure et les dommages engendrés par les radiations UV. La Figure 3 met en évidence la différence de rugosité entre une surface non exposée et exposée. Le centre de l'échantillon a été exposé à des rayonnements UV alors que le contour en a été protégé. La rugosité, RMS (roughness mean square) calculée sur une surface de $164 \mu \mathrm{m} \times 164 \mu \mathrm{m}$, est d'environ $0,1 \mu \mathrm{m}$ pour les surfaces non exposées, $0,8 \mu \mathrm{m}$ et $1,2 \mu \mathrm{m}$ pour respectivement les surfaces exposées 2 et 4 semaines. A titre de comparaison, Rosu et al. a exposé des échantillons de vinylester autour du même spectre d'émission et à la même température mais dont le flux reçu est deux fois plus important $\left(300 \mathrm{~W} / \mathrm{m}^{2}\right)$ et a également remarqué que la variation de l'indice de brillance des micrographies montrent l'augmentation de la rugosité après $200 \mathrm{~h}$ d'irradiation, alors que l'échantillon non-irradié présente une surface lisse (Rosu et al., 2008).

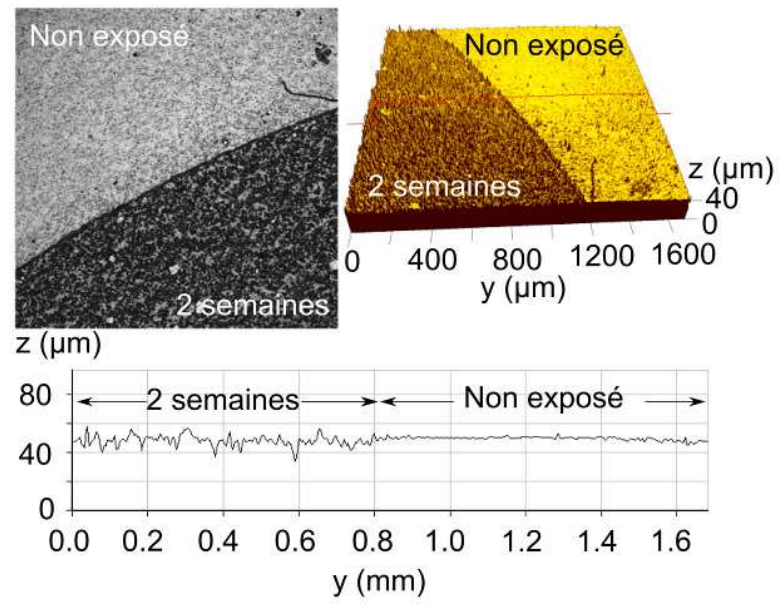

Figure 3. Micrographie LCSM 2D et 3D sur un échantillon en parti exposé pendant deux semaines et en partie protégé par l'exposition UV. Le diagramme permet de caractériser la rugosité.

L'évolution de la surface est particulièrement mise en valeur au niveau des particules additives ou des fibres. En effet, on peut remarquer que lors de l'irradiation, la matrice «s'effondre » laissant apparents les additifs et fibres qui, eux, restent intacts (Figures 4). La hauteur entre les fibres intacts et la matrice est négligeable pour une surface non exposée, de l'ordre de $5 \mu \mathrm{m}$ pour un échantillon exposé 2 semaines et de $10 \mu \mathrm{m}$ pour un échantillon exposé 4 semaines. Ces observations ont été faites sur des zones à forte, moyenne et faible densité de fibre ainsi que sur du vinylester monolithique, en évaluant la hauteur entre les particules additives et la matrice: les résultats sont comparables. Ceci signifie que l'effondrement de la matrice est indépendant de la densité des fibres. 

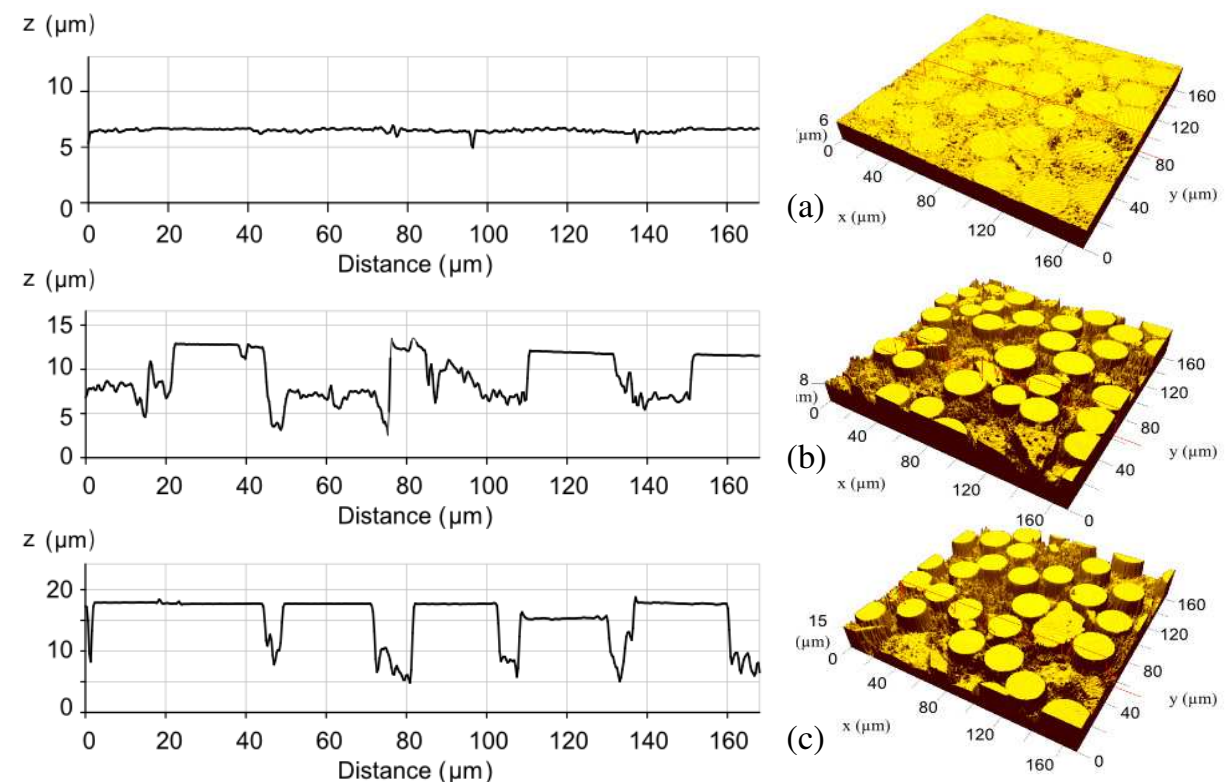

Figures 4. Micrographie 3D d'échantillons de composites (a) non exposé, (b) exposé 2 semaines et (c) exposé 4 semaines. Caractérisation de l'effondrement de la matrice à coté des fibres.

Deux hypothèses peuvent être envisagées. Premièrement, le retrait croit avec la durée d'exposition et on peut donc supposer que la matière est éliminée par ablation par suite du rayonnement UV. Elle pourra donc être confirmée ou infirmée en exposant des échantillons à de plus longues durées. Ce travail est en cours actuellement. La seconde hypothèse suppose une forme de réarrangement structural de la matrice, suivie par une contraction, suite à l'exposition aux UV, sous température. Une étude structurale appropriée est actuellement à l'étude.

\subsection{Indentation directe sur surface exposée}

Tout en gardant à l'esprit que la profondeur d'indentation est du même ordre de grandeur que la rugosité des surfaces exposées, une première étude sur une indentation directe permet, dans un premier temps, de comparer les propriétés obtenues avec des matériaux similaires précédemment étudiés. La première partie du Tableau 2 montre les résultats numériques des essais réalisés sur le vinylester monolithique; l'ordre de grandeur des résultats est comparable avec les études précédentes de Signor sur la nanoindentation du vinylester, exposé au rayonnement UV qui obtient des valeurs comprises entre 1.23 et $3.7 \mathrm{GPa}$ (Signor et al., 2003). 
On peut ensuite remarquer que les écarts-types des résultats sur les échantillons exposés (Tableau 2, en gras) sont loin d'être négligeables et ne permettent pas de conclure sur l'évolution des propriétés mécaniques du matériau. En effet, certaines hypothèses de départ ne sont pas valables dans notre cas, en particulier car la surface possède une rugosité qui crée inévitablement des déviations par rapport à l'hypothèse de contrainte hydrostatique. De plus, le vinylester étudié contient des additifs minéraux de durcissement. Pour contourner ce problème il est envisageable d'identifier sur chacune des indentations l'interaction ou non d'un additif à l'aide du LSCM. Il faut préciser que les indentations directement réalisés sur les additifs ne sont pas prises en considération dans ces résultats et les suivants également. En revanche le problème de la rugosité ne peut être directement contourné car il est impossible de la supprimer par polissage au risque d'éliminer la couche résultant des radiations UV que nous souhaitons justement caractériser.

\begin{tabular}{|c|c|c|}
\cline { 2 - 3 } \multicolumn{1}{c|}{} & $\begin{array}{c}\text { Vinylester exposé } \\
\text { monolithique }\end{array}$ & $\begin{array}{c}\text { Vinylester exposé dans } \\
\text { le composite }\end{array}$ \\
\hline Module & $5 \mathrm{GPa}$ & $10 \mathrm{GPa}$ \\
\hline Dureté & $0,25 \mathrm{GPa}$ & $0,5 \mathrm{GPa}$ \\
\hline
\end{tabular}

Tableau 1. Valeurs moyennes de module d'Young local et de rigidité estimés à partir d'essais de nanoindentation sur du vinylester exposé.

Sur la matrice vinylester dans un matériau composite exposé aux rayons UV, les caractéristiques mécaniques évaluées à partir d'indentations sont plus élevées que pour du vinylester monolithique (Tableau 1). Cette augmentation peut être expliquée par les fibres avoisinantes qui perturbent le champ de contrainte - déformation. Ce résultat peut être également comparé aux résultats de Goel qui remarque que l'évolution du module d'Young dans les régions exposées du polypropylène (PP) est plus importante dans le composite que dans le PP exposé (Goel, 2008). Il suppose que les additifs (y compris les fibres continues), accélèrent l'endommagement de la matrice.

\subsection{Analyses statistiques}

Afin de pouvoir exploiter les résultats expérimentaux, nous nous sommes inspirés de la démarche proposée par Bouzakis. Cet auteur trace la distribution statistique, à force constante, du nombre de mesures pour chaque tranche de pénétration maximale (Bouzakis, 2003). Dans notre cas, pour chaque spot, 40 mesures sont programmées (ce qui correspond au quadruple des tests précédents). Contrairement au travail de Bouzakis, les indentations sont contrôlées en déplacement et non en force. C'est donc les tranches de forces maximales qui sont étudiées. Si on considère que la rugosité influence directement la profondeur de 
pénétration, comme le suggère l'article de Bouzakis, la charge étant proportionnelle au carré de la profondeur, la rugosité influence la racine carrée de la charge. On retrouve, en effet, des résultats donnant une distribution de Gauss. Ceci constitue une avancée intéressante. En revanche, cette méthode, ne permet pas de tirer des conclusions très précises. Ce n'est dont pas la meilleure méthode à exploiter pour continuer cette étude.

\subsection{Indentation sur la section transversale}

La deuxième solution consiste à couper transversalement l'échantillon en vinylester, de l'enrober d'époxy et de le polir sur sa section transversale, dans le but d'indenter sur toute une rangée à travers l'épaisseur de l'échantillon (Figure 5). Cette méthode est directement inspirée du travail de Forster, dont la rangée d'indentation permet la caractérisation d'un revêtement multicouche (Forster, 2009). Ainsi, dans notre cas, le polissage peut être envisagé sur la tranche, tout en évitant de détruire la couche irradiée. De plus, cette méthode permet également de connaître la profondeur de l'impact des radiations UV.

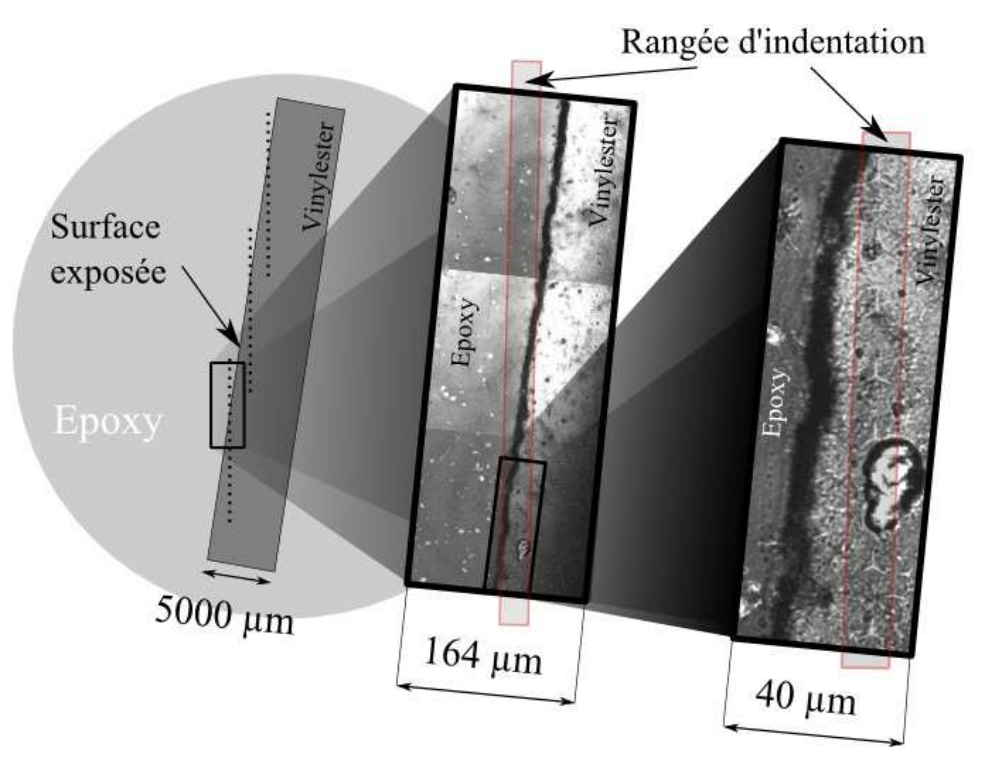

Figure 5. De gauche à droite. Le schéma représente l'échantillon de vinylester coupé sur la tranche et enrobé dans de l'époxy avec les trois rangées d'indentation opérées à travers la surface exposée. Un zoom représentant les 3 micrographies superposées identifie une rangée d'indentation sur l'époxy et le vinylester. Enfin un autre zoom est effectué pour observer les indentations sur la résine de vinylester. On peut remarquer que certaines indentations sont perturbées par une particule, ces valeurs sont évidement retirées de nos résultats. 
Deux types d'essais ont permis 2 analyses différentes: les échantillons sont placés avec un angle d'environ $10^{\circ}$ et $20^{\circ}$, entre la surface exposée et la rangée d'indentations. Pour l'angle de $10^{\circ}, 60$ indentations (dont environ $30 \%$ dans l'epoxy et $70 \%$ dans la vinylester) sont pratiquées tous les $15 \mu \mathrm{m}$. Pour l'angle de $20^{\circ}$, cinquante indentations sont réalisées tous les de $40 \mu \mathrm{m}$. Dans le premier cas (Figures 6), l'épaisseur caractérisée ainsi que l'espace entre chaque indentation sont relativement faibles et permettent d'obtenir le comportement le plus continu possible, cela permet ainsi de déterminer l'existence ou non d'un effet de bord. Dans le second cas (Figures 7), l'épaisseur caractérisée est plus importante, ce qui permet d'étudier l'évolution des propriétés mécaniques à travers l'épaisseur, notamment d'évaluer la couche affectée par l'irradiation aux rayons UV.
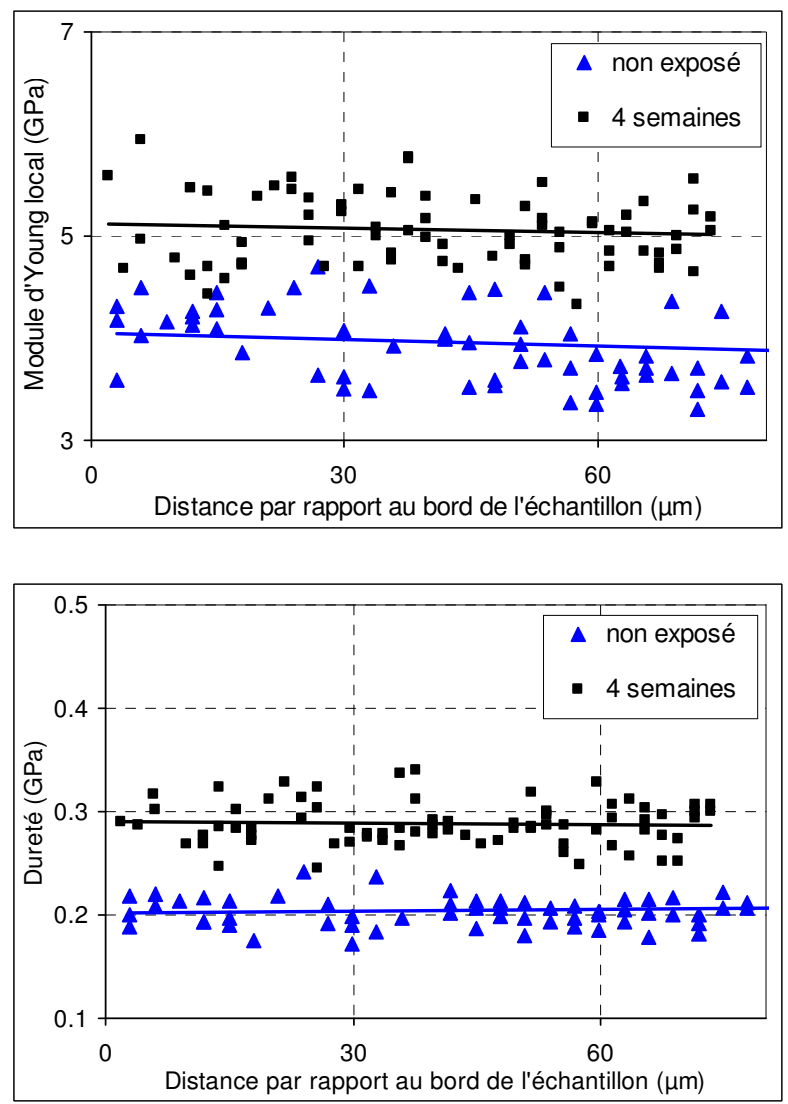

Figures 6. Module d'Young local et dureté en fonction de la distance par rapport à la surface exposée pour un échantillon non exposé et exposé 4 semaines. Utilisation de la méthode CSM et d'un indenteur de Berkovich. Les courbes de tendances sont des interpolations linéaires. 


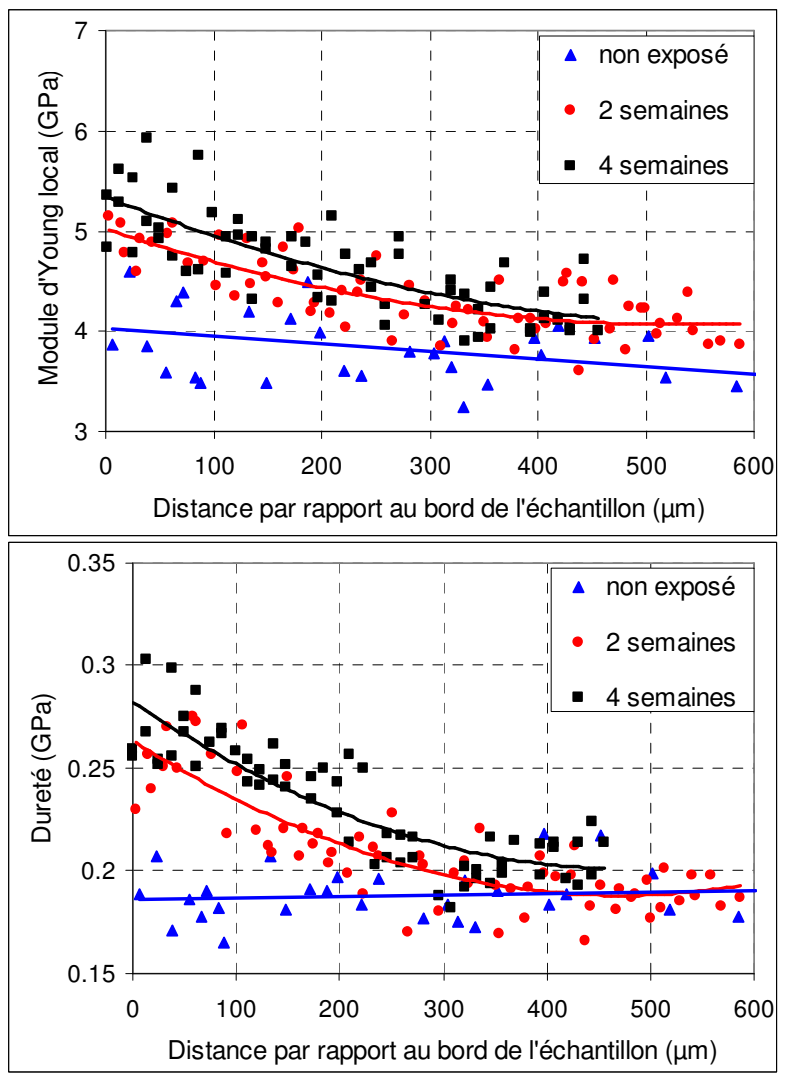

Figures 7. Module d'Young local et dureté en fonction de la distance par rapport à la surface exposée pour un échantillon non exposé et exposé 4 semaines. Utilisation de la méthode CSM et d'un indenteur de Berkovich. Les courbes de tendances sont des interpolations polynomiales (linéaire pour la surface non exposée, et de degré 2 pour les surfaces exposées).

En ce qui concerne la matrice vinylester non exposée, dans les 2 cas, les propriétés mécaniques sont quasi-constantes. Ainsi, nous pouvons supposer que le matériau est homogène et que les effets de bords sont négligeables. Cette dernière hypothèse est ainsi transposée sur les échantillons exposés.

Proche de la surface de l'échantillon (pour des valeurs d'abscisse faibles), plus le temps d'exposition est important, plus les propriétés mécaniques sont élevées et ces dernières diminuent avec la profondeur dans l'échantillon jusqu'à tendre vers des paliers correspondant aux caractéristiques du vinylester non exposé : un module 
d'Young local de 3,8 GPa et une dureté de 0,19 GPa. Ceci permet d'évaluer la couche affectée par les radiations UV à $500 \mu \mathrm{m}$. Au delà de cette limite, le matériau ne semble pas être modifié. Goel a fait les mêmes conclusions avec l'analyse de l'évolution du module d'Young, sur un échantillon de polyporpylene (PP), exposé aux radiations $\mathrm{UV}$.

\begin{tabular}{|c|c|c|c|c|c|c|c|c|}
\hline & \multicolumn{4}{|c|}{$\begin{array}{l}\text { Indentation directe : } \\
\text { dégradation-UV sur la surface }\end{array}$} & \multicolumn{4}{|c|}{$\begin{array}{c}\text { Section transversale : rugosité } \\
\text { polie }\end{array}$} \\
\hline & \multicolumn{2}{|c|}{ Module (GPa) } & \multicolumn{2}{|c|}{ Dureté (GPa) } & \multicolumn{2}{|c|}{ Module (GPa) } & \multicolumn{2}{|c|}{ Dureté (GPa) } \\
\hline & Moy. & $\begin{array}{l}\text { Écart } \\
\text { type }\end{array}$ & Moy. & $\begin{array}{l}\text { Écart } \\
\text { type }\end{array}$ & Moy. & $\begin{array}{l}\text { Écart } \\
\text { type }\end{array}$ & Moy. & $\begin{array}{l}\text { Écart } \\
\text { type }\end{array}$ \\
\hline Non exposé & 4,58 & 0,66 & 0,231 & 0,011 & 3,84 & 0,35 & 0,189 & 0,014 \\
\hline 2 semaines & 4,40 & 2,39 & 0,123 & 0,119 & 4,36 & $\mathbf{0 , 3 7}$ & 0,209 & $\mathbf{0 , 0 2 7}$ \\
\hline 4 semaines & 4,39 & 1,52 & 0,132 & 0,091 & 4,65 & 0,48 & 0,232 & $\mathbf{0 , 0 3 0}$ \\
\hline
\end{tabular}

Tableau 2. Moyenne (Moy.) et écart type des résultats de dureté et module d'Young local estimé par nanindentation sur du vinylester seul avec la méthode CSM et un indenteur de Berkovich.

Sans prendre en compte la décroissance des propriétés mécaniques, on remarque déjà que l'écart type des résultats de nanoindentation sur la surface transversale est beaucoup plus faible que sur les essais directement réalisés sur la surface escarpée. Il existe néanmoins toujours des écarts sur les résultats. Ces derniers peuvent être expliqués par la localisation des indentations dans le proche voisinage des additifs minéraux. En effet, même si l'indentation n'est pas effectuée directement sur un additif, il est possible que la zone sous l'indenteur soit altérée par une particule présente au alentour de l'indentation (Figure 8).

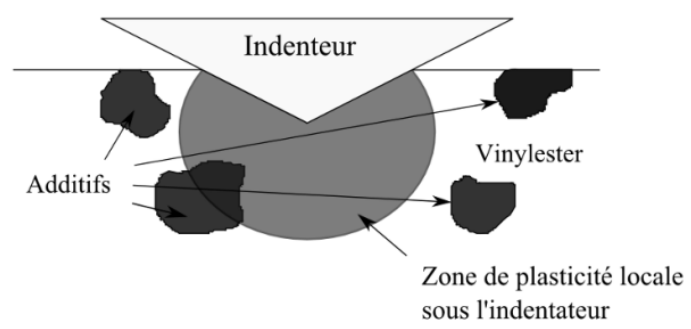

Figure 8. Indentation sur une surface sans additifs mais dont la zone de plasticité locales est affectée par les particules environnantes. 


\section{Conclusion}

Les images obtenues par le microscope confocal nous permettent de remarquer «l'effondrement» de la matrice par rapport aux additifs et aux fibres qui restent intacts. Des expériences complémentaires, en augmentant le temps d'exposition, permettraient de définir s'il s'agit d'une ablation du matériau ou d'une modification de structure de la matrice. Ces études sont en cours de réalisation.

Les très importantes déviations des résultats obtenues à partir des indentations réalisées directement sur la surface exposée sont dues à la rugosité engendrée par la dégradation suite à l'irradiation aux UV. C'est pourquoi l'indentation «directe » sur la surface est inexploitable, même en utilisant une analyse statistique qui s'avère peu précise. La solution envisagée consiste donc à couper l'échantillon transversalement, l'enrober, le polir et d'opérer une rangée d'indentations à travers la profondeur. La dispersion des résultats étant beaucoup plus faible, cela nous permet de conclure que cette méthode est beaucoup plus fiable. Les résultats montrent que le module d'Young local et la dureté sont affectés sur une épaisseur de $0.5 \mathrm{~mm}$ et que ces caractéristiques mécaniques augmentent avec la durée d'exposition.

\section{Remerciement}

Ce travail a été conduit dans le cadre du projet FUI (fonds uniques interministériels de la DGE), Decid2. Les auteurs tiennent à remercier ces fonds, ainsi que la région Pays de la Loire pour le soutien financier.

\section{Bibliographie}

K.-D. Bouzakis, "The effect of specimen roughness and indenter tip geometry on the determination accuracy of thin hard coatings stress-strain laws by nanoindentation", Materials characterization, Vol. 49, 149-156, 2003

S. W. Brown, G P Eppeldauer, K R Lykke « NIST facility for Spectral Irradiance and Radiance Responsivity Calibrations with Uniform Sources ». Metrologia, Vol. 37, pp. $579,2000$.

Robert F. Cook, Michelle L. Oyen, "Nanoindentation behavior and mechanical properties measurement of polymeric materials", Int. J. Mat. Res. (formerly Z. Metallkd), Vol. 98, 370-378, 2007

Monssef Drissi-Habti, Kikuo Nakano, "Local Mechanical Characterisation and Modelling of the Interfacial Behaviour In Hi-Nicalon/BN/ $\alpha-S i 3 N 4$ Ceramic Matrix Composites by Way of Instrumented Microindentatin Tests". Journal of the European Ceramic Society, Vol. $18,1845-1855,1998$

Anthony C. Fischer-Cripps, "Nanoindentation, Second Edition”, Springer, 260p, 2004

Aaron M. Forster, « Modulus and Chemical Mapping of Multilayer Coating ». ACS Appl. Mat. Interfaces, Vol. 3, 597-603, 2009 
A. Goel, "Effect of UV exposure on the microstructure and mechanical properties of long fiber thermoplastic (LFT) composites", J Mater Sci, Vol. 43, 4423-4432, 2008

C. S. Helbling and V.M. Karbhari, "Investigation of the Sorption and Tensile Response of Pultruded E-Glass/Vinylester Composites Subjected to Hygrothermal Exposure and Sustain Strain", Journal of reinforced plastics and composites, Vol. 27, page 613, 2008

Martin, J.W., Nguyen, T., and Wood, K.A., "Unresolved Issues Related to Predicting the Service Life of Polymeric Materials," Service Life Prediction: Challenging the Status Quo, Martin, J.W., Ryntz, R.A., and Dickie, R.A. (Eds.), Federation of Societies for Coatings Technology, Blue Bell, PA, p. 13, 2005.

W. C. Oliver G.M. Pharr, «An improved technique for determining hardness and elastic modulus using load and displacement sensing indentation experiments ». Materials Research Society, Vol. 7, No. 6, 1564-1583, Juin 1992

Dans Rosu, Liliana Rosu, Constantin N. Cascaval, "Effect of ultraviolet radiation on vinyl ester network based on bisphenol A", Journal of Photochemistry and Photobiologu A : Chemistry, 194, 275-282, 2008

Andrew W. Signor, Mark R. VanLandingham, Joannie W. Chin, "Effects of ultraviolet radiation exposure on Vinylester resins: characterization of chemical, physical and mechanical damage”, Polymer Degradation and Stability, Vol. 79, 359-368, 2003 